\title{
Reação anafilática ao corante azul patente durante a biópsia do linfonodo sentinela em câncer de mama inicial - relato de caso
}

\author{
Anaphylactic reaction to patent blue dye during sentinel \\ lymph node biopsy in early-stage breast cancer - a case report
}

Julia Yoriko Shinzato ${ }^{1}$, Ana Carolina Pereira Marcaccini² ${ }^{2}$, Angélica de Fátima de Assunção Braga ${ }^{3}$, Arneth Rodrigues Ribeiro ${ }^{4}$, José Eduardo Marco Rubio ${ }^{5}$, João Paulo Dias de Souza ${ }^{6}$

RESUMO

A biópsia do linfonodo sentinela no tratamento do câncer de mama vem substituindo a linfadenectomia em casos iniciais. Este tratamento pode ser realizado com o corante azul vital e/ou radiofármaco. Há relatos de reações alérgicas ao corante com diferentes graus de severidade. Relata-se um caso de reação anafilática severa após injeção intradérmica do corante azul patente, em paciente com diagnóstico de carcinoma ductal in situ. O corante azul patente, apesar de facilitar a detecção do linfonodo, apresenta risco de desencadear reações anafilactóides. É necessário que a equipe envolvida tenha preparo para diagnosticar e tratar esta complicação.

PALAVRAS-CHAVE: Anafilaxia/induzido quimicamente; Hipersensibilidade; Agentes corantes/efeitos adversos; Biópsia de linfonodo sentinela/efeitos adversos; Neoplasias mamárias/patologia; Carcinoma intraductal não infiltrante/cirurgia; Relatos de casos [Tipo de publicação]

\section{ABSTRACT}

Sentinel lymph node biopsy in the treatment of breast cancer has been replacing lymph node resection in early cases. This treatment may be performed with blue dye and/or radiopharmaceuticals. There are reports of allergic reactions to blue dye with different degrees of severity. A case of severe anaphylactic reaction after intradermal injection of patent blue dye was reported in a patient diagnosed with ductal carcinoma in situ. The patent blue dye facilitates the detection of the sentinel lymph node, but there is the risk of triggering anaphylactic reactions. It is recommended the team involved to be very knowledgeable and prepared to diganose and treat this complication.

KEYWORDS: Anaphylaxis/chemically induced; Hypersensitivity; Coloring agents/adverse effects; Sentinel lymph node biopsy/adverse effects; Breast neoplasms/pathology; Carcinoma, intraductal, noninfiltrating/surgery; Case reports [Publication type]

1 Professora do Departamento de Tocoginecologia da Faculdade de Ciências Médicas da Universidade Estadual de Campinas - UNICAMP - Campinas (SP), Brasil.

2 Residente de Mastologia do Departamento de Tocoginecologia da Faculdade de Ciências Médicas da Universidade Estadual de Campinas - UNICAMP - Campinas (SP), Brasil.

3 Professora Associada do Departamento de Anestesiologia da Faculdade de Ciências Médicas da Universidade Estadual de Campinas - UNICAMP - Campinas (SP), Brasil.

4 Médica Associada da Divisão de Anestesiologia do Centro de Atenção Integral à Saúde da Mulher (CAISM) da Faculdade de Ciências Médicas da Universidade Estadual de Campinas - UNICAMP - Campinas (SP), Brasil.

5 Médico Associado da Área de Oncologia do Centro de Atenção Integral à Saúde da Mulher (CAISM) da Faculdade de Ciências Médicas da Universidade Estadual de Campinas - UNICAMP - Campinas (SP), Brasil.

6 Médico Associado da Unidade de Terapia Intensiva do Centro de Atenção Integral à Saúde da Mulher (CAISM) da Faculdade de Ciências Médicas da Universidade Estadual de Campinas - UNICAMP - Campinas (SP), Brasil.

Correspondência: Julia Yoriko Shinzato

Rua Proença, 991, apto. 43 - 13026-121 - Campinas - SP - Fone/Fax: (19) 32311068 - e-mail: jshinzato@mpcnet.com.br

Recebido em: 24/8/2006

Aceito com modificaçōes em: 27/11/2006

Rev Bras Ginecol Obstet. 2006; 28(12): 728-32. 


\section{Introdução}

A biópsia do linfonodo sentinela no tratamento cirúrgico do câncer de mama inicial vem sendo amplamente empregada como parte do protocolo de rotina, evitando a linfadenectomia total na maioria dos casos. Para a identificação do linfonodo sentinela podem ser utilizados o corante azul vital ${ }^{1}$ ou o radiofármaco tecnécio ${ }^{2}$, ambos de forma isolada ou em associação. A associação dessas duas modalidades apresenta sensibilidade de $98 \%$ na identificação do linfonodo sentinela. No entanto, recentemente vêm sendo descritas reações de hipersensibilidade ao corante azul, numa incidência que varia de 0,1 a $2 \%$, e alguns casos classificados como severos cursam com repercussões hemodinâmicas e necessidade de drogas vasoativas ${ }^{3-10}$. No serviço de mastologia do Centro de Atenção Integral a Saúde da Mulher (CAISM) da Universidade Estadual de Campinas (UNICAMP), o emprego da técnica de biópsia do linfonodo sentinela teve início em março de 2004, utilizando a associação dos dois métodos. O objetivo deste relato é apresentar um caso de reação alérgica no período intra-operatório após a injeção intradérmica de corante azul vital.

\section{Relato do caso}

Paciente com 47 anos procurou o ambulatório de Mastologia do CAISM da UNICAMP, com queixa de nódulo doloroso e de crescimento progressivo na mama esquerda há três meses. Não possuía antecedente familiar de doenças oncológicas. De antecedentes pessoais, referiu NIC III do colo do útero, tratado com cirurgia de alta freqüência sob anestesia local. Ao exame físico ginecológico, apresentava nódulo de $1,5 \mathrm{~cm}$ na região do prolongamento axilar esquerdo, endurecido, pouco aderido a planos profundos, sem alteração de pele e aumento da densidade do parênquima na região do QSE, com difícil individualização de nódulos.

A mamografia mostrava calcificações pleomórficas com áreas de agrupamento comprometendo QSE e pequena porção do QSI da mama esquerda (BIRADS 5); o exame ultra-sonográfico da mama esquerda evidenciou área suspeita, mal delimitada, heterogênea, com calcificações de 32 x 19 x 20 mm no QSE. O exame anátomo-patológico realizado sob core biópsia mostrou carcinoma ductal in situ, GN 3, tipo comedo com extensão lobular multifocal. Foi indicada mastectomia com biópsia do linfonodo sentinela. Na avaliação pré- anestésica, a paciente apresentava-se em bom estado geral, corada, hidratada, sem antecedentes de doenças cardiovasculares, respiratórias, endócrinas e história de alergia; no eletrocardiograma: ritmo sinusal; exames laboratoriais normais, tabagista, classificada como estado físico ASA 2.

Cinco horas antes da cirurgia foi administrado 0,4 mCi de fitato marcado com tecnécio $99 \mathrm{mtc}$, via intradérmica na região periareolar da mama esquerda, evidenciando a captação de um linfonodo sentinela por meio do exame de linfocintilografia. A cirurgia foi realizada sob anestesia geral com ventilação controlada mecânica. Como medicação pré-anestésica foi utilizado midazolam $(7 \mathrm{mg}$ por via intramuscular) 30 minutos antes do início da anestesia. Na sala cirúrgica, procedeu-se a venóclise no membro superior D com cânula $18 \mathrm{G}$ para reposição volêmica e administração de drogas. A monitorização constou de cardioscópio (derivação DII), medida não-invasiva de pressão arterial, oximetria de pulso, capnografia e avaliação do bloqueio neuromuscular por aceleromiografia.

Iniciou-se a indução da anestesia geral com pré-oxigenação com $\mathrm{O}_{2}$ a 100\% sob máscara, injeção venosa de sufentanil (30 $\mu \mathrm{g})$, propofol (120 mg) e rocurônio (50 mg), seguida de intubação traqueal. A manutenção da anestesia foi obtida com isoflurano em concentração de 1 a $2,0 \%$ por meio de vaporizador calibrado, veiculado em mistura de $\mathrm{O}_{2}$ e $\mathrm{N}_{2} \mathrm{O}(50 \%)$. Os parâmetros cardio-respiratórios iniciais eram: volume corrente $(600 \mathrm{~mL})$, freqüência respiratória $(12 \mathrm{rpm})$, saturação periférica de $\mathrm{O}_{2}(98 \%)$, PA (110x50 $\left.\mathrm{mmHg}\right), \mathrm{CO}_{2}$ expirado (26 mmHg). Seguiu-se antibiótico-profilaxia (cefazolina $2 \mathrm{~g}$ ) diluída em Ringer com lactato (500 mL) em infusão continua; assepsia e antissepsia com PVPI, injeção intra-dérmica de azul patente $(1 \mathrm{~mL})$.

Após 15 minutos da injeção do corante azul patente, já tendo iniciado a cirurgia, a paciente apresentou um declínio progressivo da saturação de oxigênio (de 97 para 88\%) e da pressão arterial sistólica (100 para 60 e $40 \mathrm{mmHg}$ ), sem alterações da freqüência cardiaca $(70 \mathrm{bpm})$, de ausculta pulmonar e de pressão de $\mathrm{CO}_{2}$ expirado. Neste momento foi aventada a hipótese de reação anafilática reforçada pela visualização de rash cutâneo nas pálpebras, interrompendo-se a administração dos agentes anestésicos. Manteve-se a ventilação com oxigênio a $100 \%$ sob pressão positiva, aumentou-se a infusão de cristalóides, administrou-se efedrina (25 mg), sem eficácia; seguiu-se injeção de adrenalina em doses fracionadas $(0,1 \mathrm{mg})$, lentamente por via endovenosa (total de $1 \mathrm{mg}$ ) para tratamento de hipotensão arterial e manutenção da pressão arterial em niveis normais. Adicionalmente, empregou-se hidrocortisona (1 g) e difeni- 
dramina $(50 \mathrm{mg})$. Diante do quadro, optou-se por interrupção do procedimento.

A paciente foi admitida na UTI sedada (Ramsay 6), sob ventilação mecânica, corada, hidratada, pupilas mióticas e manutenção de pressão arterial com drogas vasoativas. Encontrava-se bradicárdica e com roncos esparsos à ausculta pulmonar, sem alterações na perfusão. Evoluiu com tendência à hipotensão arterial, apesar da infusão de soluções cristalóides e colóides (2000 mL), sendo necessário o uso de noradrenalina, com doses tituladas entre 0,05 e $0,20 \mu \mathrm{g} \mathrm{kg}{ }^{-1} \mathrm{~min}$.

Após as primeiras 14 horas de pós-operatório, houve estabilização hemodinâmica, sendo possivel a retirada gradual da noradrenalina. Neste periodo, a paciente foi mantida em ventilação mecânica sob sedação contínua com midazolam e fentanil e extubada na $18^{a}$ hora de pós-operatório, com alta no dia seguinte. Solicitada interconsulta com a Disciplina de Imunologia, foi recomendada dessensibilização com prednisona. Após o esquema de corticoterapia, a paciente foi submetida a mastectomia com linfadenectomia axilar dos niveis I e II, empregando-se a mesma técnica anestésica utilizada em cirurgia anterior, e evoluiu sem intercorrências. Apresentou um período pós-operatório normal na enfermaria e recebeu alta em bom estado. O exame anátomo-patológico definitivo mostrou carcinoma ductal invasivo multifocal e todos os linfonodos eram negativos para metástase.

\section{Discussão}

A biópsia do linfonodo sentinela apresentase atualmente como a abordagem cirúrgica mais conservadora em casos de câncer invasivo inicial, evitando-se a linfadenectomia total em presença de linfonodos livres de comprometimento neoplásico, sendo este procedimento também indicado em alguns casos de carcinoma in situ extenso com indicação de mastectomia ${ }^{10}$. Severas reações anafilactóides ao corante azul necessitando de ressuscitação, embora raras, têm sido descritas em pacientes com carcinoma de mama submetidas à biópsia do linfonodo sentinela. Essas reações podem ocorrer em graus variáveis de intensidade, podendo ser classificadas desde leves, com apenas eritema cutâneo, até reação anafilática severa, com hipotensão arterial e necessidade de drogas vasoativas $^{5}$. No serviço de Mastologia do CAISM da UNICAMP, a técnica da biópsia do linfonodo sentinela teve início em março de 2004, tendo sido realizadas até abril de 2006 um total de 132 casos, utilizando a combinação do radiofármaco e do corante azul patente. Desses, em apenas um caso $(0,7 \%)$ ocorreu reação anafilática severa após a injeção do corante azul patente, incidência semelhante à relatada na literatura, que varia de 0,1 a $1,1 \%{ }^{4,11}$.

Apesar da alta sensibilidade apresentada pelo uso isolado do radiofármaco (98\%), sua associação com o corante azul facilita a identificação do linfonodo sentinela durante o ato operatório, por meio da visualização direta do linfonodo corado de azul ${ }^{2}$.

Os corantes mais utilizados para a identificação do linfonodo sentinela são azul isossulfan e o azul patente, e, numa menor freqüência, o azul de metileno. Tanto o azul isossulfan como o azul patente pertencem ao grupo de corantes triarilmetano, tendo o azul patente apenas um grupo hidroxila adicional ${ }^{6}$. Têm sido atribuídas aos corantes inúmeras reações de hipersensibilidade imediatas. Ao contrário, o azul de metileno tem sido considerado mais seguro do que o azul patente, constituindo uma alternativa efetiva a esse, na identificação de linfonodo sentinela em mulheres com câncer de mama, fato este contestado por alguns autores que relatam casos de hipersensibilidade também ao azul de metileno ${ }^{7,12,13}$. Mullan et al. ${ }^{8}$ acreditam que cerca que $2,7 \%$ da população seria alérgica ao corante azul, mesmo na ausência de história prévia de alergia ou testes cutâneos negativos, o que pode ser atribuído à sensibilização ocasionada pela exposição repetida a alguns produtos como tecidos, cosméticos, papel, couro e medicamentos, que contém estes corantes ${ }^{5}$.

O azul patente, após injeção intradérmica ou intraparenquimatosa, é captado pelos vasos linfáticos da área de drenagem, sendo 2/3 da droga absorvida na primeira hora e completamente em 24 horas. É excretado pela urina (10\%) e o paciente pode apresentar urina de coloração azul por até 24 horas $^{11}$.

Neste presente caso, a paciente não teve nenhum contacto prévio conhecido com o azul patente; no entanto, é possivel que apresentasse sensibilização devido ao uso de produtos que contenham esses corantes. As alterações hemodinâmicas e ventilatórias, assim como as reações cutâneas sugestivas de reação alérgica ao azul patente, foram evidenciadas aos 15 minutos após a injeção do corante azul patente, tempo similar ao relatado na literatura e que varia de 8 a 45 minutos $^{5}$.

O mecanismo de ação envolve o desenvolvimento do anticorpo imunoglobulina específico IgE; durante a exposição, o antígeno induziria a degranulação destas células, levando a um aumento de histamina e mediadores vasoativos da anafilaxia ${ }^{11}$. Reações alérgicas durante procedimentos anestésico-cirúrgicos também podem ser atribuídas em 60$80 \%$ dos casos aos bloqueadores neuromusculares, 
sendo, em 50\% destes, reação do tipo IgE mediado. A possibilidade de se atribuir a reação alérgica ao rocurônio, bloqueador neuromuscular empregado neste caso, foi descartada, uma vez que estas reações ocorrem quase que imediatamente após a injeção da droga ${ }^{12}$. Adicionalmente, esta paciente foi submetida em outro tempo a mastectomia sob anestesia geral com as mesmas drogas utilizadas em procedimento anterior, sem apresentar qualquer intercorrência.

Como manifestações clínicas da reação alérgica ao azul patente, são descritas alterações cutâneas como urticária, pápulas azuis, eritema e placas difusas maculo-papulares, acompanhadas ou não de colapso circulatório, broncoespasmo e diminuição na saturação de oxigênio, sinais dominantes de reação severa ${ }^{3,14}$. Piñero et $a{ }^{15}$ relatam a ocorrência de redução no nivel de saturação de oxigênio avaliada pela oximetria do pulso em todas as pacientes que utilizaram algum tipo de corante azul. Entretanto, não encontraram uma correspondência à real dessaturação quando avaliada pela gasometria arterial. Neste caso, embora a reação cutânea tenha sido leve e localizada, observou-se rápida e acentuada dessaturação de $\mathrm{O}_{2}$, associada a hipotensão severa de difícil tratamento com drogas vasoativas. Não ocorreu broncoespasmo, um sintoma mais raro do quadro, atribuído à liberação local de mediadores broncoconstritivos ${ }^{12}$. Conforme recomendado na literatura, o tratamento constou da administração de oxigênio a 100\%, infusão venosa rápida de fluidos, antihistamínicos, epinefrina e glicocorticóides ${ }^{4,11,14}$. Em um único trabalho, relata-se a ocorrência de nova reação anafilática após tratamento da primeira reação, levando a decisão da equipe pela realização da mastectomia radical com intuito de remover todo o corante residual ${ }^{14}$

Alguns autores recomendam a profilaxia préoperatória com administração de glicocorticóide, difenidramina e famotidina, pois foi demonstrado que tal medida reduz o grau da severidade da reação; entretanto, sem alterar a incidência geral ${ }^{16}$. Outros recomendam o uso profilático de difenidramina e ranitidina como bloqueador antihistamínico de receptores $\mathrm{H} 1$ e $\mathrm{H} 2^{5}$. Entretanto, há trabalhos que contestam o uso rotineiro da profilaxia, alegando ser baixa a incidência da reação anafilática severa e que ainda mais estudos sobre a real eficácia dessas medidas seriam necessários ${ }^{4}$.

Outra questão também ainda controversa é como confirmar o diagnóstico de reação anafilática ao corante azul. Sandhu et $\mathrm{al}^{17}$ evidenciaram altos niveis de triptase sanguínea, entretanto, esta dosagem não é sensivel, nem específica, podendo estar falsamente elevada em pacientes com niveis basais elevados. Outra possibilidade é o emprego de teste cutâneo ou intradérmico; no entanto, ambos podem não apresentar positividade ${ }^{6-8}$.

De acordo com a revisão da literatura, a redução progressiva da saturação de oxigênio constitui o primeiro sinal de reação anafilática ao corante azul, embora Piñero et al. ${ }^{15}$ tenham relatado, em todos os casos do uso do corante azul, leve dessaturação, sem necessariamente significar reação anafilática. Contudo, a diminuição da saturação de oxigênio, acompanhada ou não de reações cutâneas e hipotensão arterial, requer a instituição de rápido tratamento, com oxigênio a 100\%, infusão de líquidos, administração de corticóide e difenidramina, além de adrenalina nos casos mais severos. A confirmação da reação anafilática ao corante azul nem sempre é possivel com os diferentes testes descritos na literatura e o uso de medicamento profilático ainda é controverso.

Face ao exposto, durante o uso do corante azul patente na identificação do linfonodo sentinela é de fundamental importância que toda a equipe envolvida tenha conhecimento da possibilidade da ocorrência de reação anafilática e preparo adequado no reconhecimento imediato e tratamento das repercussões. Apesar de raro, o choque anafilático ao azul patente expõe a paciente ao risco iminente de vida.

\section{Referências}

1. Giuliano AE. Sentinel lymphadenectomy in primary breast carcinoma: an alternative to routine axillary dissection. J Surg Oncol. 1996;62(2):75-7.

2. Veronesi U, Paganelli G, Galimberti V, Viale G, Zurrida $\mathrm{S}$, Bedoni M, et al. Sentinel-node biopsy to avoid axillary dissection in breast cancer with clinically negative lymph-nodes. Lancet. 1997;349(9069):1864-7

3. Montgomery LL, Thorne AC, Van Zee KJ, Fey J, Heerdt AS, Gemignani M, et al. Isosufan blue dye reactions during sentinel lymph node mapping for breast cancer. Anesth Analg. 2002;95(2):385-8.

4. Amr D, Broderick-Villa G, Haig PI, Guenter JM, DiFronzo LA. Adverse drug reactions during lymphatic mapping and sentinel lymph node biopsy for solid neoplasms. Am Surg. 2005;71(9):720-4.

5. Cimmino VM, Brown AC, Szocik JF, Pass HA, Moline $\mathrm{S}$, De SK, et al. Allergic reactions to isosulfan blue during sentinel node-biopsy - a common event. Surgery. 2001;130(3):439-42.

6. Scherer K, Studer W, Figueiredo V, Bircher AJ. Anaphylaxis to isosulfan blue and cross-reactivity to patent blue V: case report and review of nomenclature of vital blue dyes. Ann Allergy Asthma Immunol. 2006;96(3):497-500. 
7. Dewachter P, Mouton-Faivre C, Trechot P, Lleu JC, Mertes PM. Severe anaphylatic shock with methylene blue instillation. Anesth Analg. 2005;101(1): 149-50.

8. Mullan MH, Deacock SJ, Quiney NF, Kissin MW. Anaphylaxis to patent blue dye during sentinel lymph node biopsy for breast cancer. Eur J Surg Oncol. 2001;27(2):218-9.

9. Crivellaro M, Senna G, Dama A, Bonadonna P, Passalacqua G. Anaphylaxis due to patent blue dye during lymphography, with negative skin prick test. J Investig Allergol Clin Immunol. 2003;13(1):71-2.

10.Schwartz GF, Giuliano AE, Veronesi U; The Consensus Conference Committee. Proceedings of the consensus conference on the role of sentinel lymph node biopsy in carcinoma of the breast, April 19-22, 2001, Philadelphia, Pennsylvania. Cancer. 2002;94(10):2542-51.

11.Albo D, Wayne JD, Hunt KK, Rahlfs TF, Singletary $\mathrm{SE}$, Ames FC, et al. Anaphylatic reactions to isosulfan blue dye during sentinel lymph node biopsy for breast cancer. Am J Surg. 2001;182(4):393-8.

12.Heir T, Guttormsen AB. Anaphylactic reactions during induction of anaesthesia using rocuronium for muscle relaxation: a report including 3 cases. Acta Anaesthesiol Scand. 2000;44(7):775-81.

13. Dewachter P, Mouton-Faivre C, Benhaijoub A, AbelDecollogne F, Mertes PM. Anaphylactic reaction to patent blue $\mathrm{V}$ after sentinel lymph node biopsy. Acta Anaesthesiol Scand. 2006;50(2):245-7

14.Efron P, Knudsen E, Hirshorn S, Copeland EM. Anaphylactic reaction to isosulfan blue used for sentinel node biopsy: case report and literature review. Breast J. 2002;8(6):396-9.

15.Piñero A, Illana J, Garcia-Palenciano C, Cañizarese F, Canteras M, Cañadillas V, et al. Effect on oximetry of dyes used for sentinel lymph node biopsy. Arch Surg. 2004;139(11):1204-7

16. Raut CP, Hunt KK, Akins JS, Daley MD, Ross MI, Singletary SE, et al. Incidence of anaphylactoid reactions to isosulfan blue dye during breast carcinoma lymphatic mapping in patients treated with preoperative prophylaxis: results of a surgical prospective clinical practice protocol. Cancer. 2005;104(4): 692-9.

17. Sandhu S, Farag E, Argalious M. Anaphylaxis to isosulfan blue dye during sentinel lymph node biopsy. J Clin Anesth. 2005;17(8):633-5. 